\title{
Neutrophil-to-lymphocyte ratio correlates with prognosis and response to chemotherapy in patients with non-M3 de novo acute myeloid leukemia
}

\author{
Qianying Zhang ${ }^{1 \#}$, Qianqian Yang ${ }^{2 \#}$, Yiqin Weng ${ }^{1}$, Ziyang Huang ${ }^{1}$, Rongrong Chen ${ }^{1}$, Yiyi Zhu ${ }^{1}$, \\ Kanchun Dai ${ }^{3}$, Shenghui Zhang ${ }^{1,2}$, Songfu Jiang ${ }^{1,2}$, Kang Yu ${ }^{1,2}$ \\ ${ }^{1}$ Department of Hematology, Wenzhou Key Laboratory of Hematology, Wenzhou, China; ${ }^{2}$ Division of Clinical Research, The First Affiliated \\ Hospital of Wenzhou Medical University, Wenzhou, China; ${ }^{3}$ Ningbo Huamei Hospital University of Chinese Academy of Sciences, Ningbo, China \\ Contributions: (I) Conception and design: Q Zhang, Q Yang; (II) Administrative support: None; (III) Provision of study materials or patients: Y Weng, \\ Z Huang, R Chen; (IV) Collection and assembly of data: Y Zhu, K Dai; (V) Data analysis and interpretation: S Jiang, S Zhang, K Yu; (VI) Manuscript \\ writing: All authors; (VII) Final approval of manuscript: All authors. \\ \#These authors contributed equally to this work. \\ Correspondence to: Songfu Jiang, MD. Department of Hematology, Wenzhou Key Laboratory of Hematology, the First Affiliated Hospital of \\ Wenzhou Medical University, Wenzhou, China. Email: jiangsongfu@189.cn; Kang Yu, MD. Department of Hematology, Wenzhou Key Laboratory \\ of Hematology, the First Affiliated Hospital of Wenzhou Medical University, Wenzhou, China. Email: yukang62@126.com.
}

\begin{abstract}
Background: Neutrophil-to-lymphocyte (NLR) ratio can predict survival outcome and assess response to chemotherapy in several tumors. However, the values of NLR in acute myeloid leukemia (AML) remains unknown.

Methods: A retrospective review of 181 patients with de novo AML excluding acute promyelocytic leukemia (M3) was conducted in our institute. We categorized the patients into two groups by defining NLR =2.0 as the cut-off point. NLR was calculated by the ratio of the number of neutrophils to lymphocytes in the peripheral blood (PB). The baseline clinicopathologic parameters were compared using Chi-squared test or Kruskal-Wallis H test. Kaplan-Meier analysis was used to assess survival, and overall survival (OS) and disease-free survival (DFS) were analyzed using the Cox regression with log-rank tests.
\end{abstract}

Results: We found AML patients with low NLR (<2.0) had longer OS and DFS than those with high NLR ( $\geq 2.0)$. NLR, absolute neutrophil count (ANC), and absolute lymphocyte count (ALC) were significantly associated with OS and DFS in all AML patients. NLR, ANC, and ALC were associated with OS and DFS only in those case with myeloblasts over $50 \%$ in bone marrow (BM). Furthermore, the median NLR was dramatically increased in low NLR group when patients achieved complete remission (CR).

Conclusions: Pretreatment NLR as a marker can predict the prognosis and NLR can assess the response to chemotherapy in patients with non-M3 AML, especially in those cases with myeloblasts over 50\% in BM.

Keywords: Acute myeloid leukemia (AML); disease-free survival (DFS); neutrophil-to-lymphocyte ratio (NLR ratio); overall survival (OS)

Submitted May 25, 2020. Accepted for publication Oct 21, 2020.

doi: $10.21037 /$ tcr-20-2179

View this article at: http://dx.doi.org/10.21037/tcr-20-2179

\section{Introduction}

Acute myeloid leukemia (AML) is characterized by a block in myeloid differentiation leading to accumulation of immature hematopoietic cells $(1,2)$. Despite advances in therapeutics and supportive care, most adult AML patients die from their disease. Most patients who diagnosed as AML treated with Idarubicin and arabinocytidine (IA) based chemotherapy, or Daunorubicin and arabinocytidine 
(DA) or homoharringtonine and arabinocytidine (HA). Therefore, it is crucial to improve the current grading system including effective diagnostic and prognostic biomarkers for risk stratification of patients and bettercustomized treatment. Our previous studies have revealed that pretreatment platelet count and plasma fibrinogen levels have predictive values for the prognosis of $\mathrm{AML}$ patients excluding acute promyelocytic leukemia (M3) $(3,4)$.

According to the NCCN guidelines, bone marrow (BM) analysis with cytogenetics and evaluation of several molecular markers, including RUNX1-RUNX1T1, CBFB-MYH11, CEBPA, FLT3-ITD, MLLT3-KMT2A, DEK-NUP214, NPM1, c-KIT and so on, are necessary to establish the diagnosis and risk assessment and prognostication for AML (5). However, the behavior of AML is unpredictable with considerably different clinical outcomes from patients with the same risk status based on validated cytogenetics and molecular abnormalities. It is well recognized that inflammation plays a key role in cancer biology (6-8). Chronic inflammation characterized by continuous production of pro-inflammatory signals may produce a maladaptive circumstance in which continued exposure to stress conditions caused by continued proliferation, BM niche dysfunction, and exposure to stressors including reactive oxygen species facilitates genomic instability and potentially the acquisition of somatic mutations $(9,10)$. Therefore, chronic inflammation may function as an initiator of hematological malignancy (9). The cancer-related inflammatory response contributes to proliferation and survival of malignant cells, subversion of adaptive immunity and reduced response to chemotherapeutic agents $(11,12)$. Neutrophils and lymphocyte as two main types of cells involved in inflammation, and peripheral neutrophil-to-lymphocyte (NLR) ratio, has been proposed as a potential prognostic factor for many solid tumors, such as hepatocellular carcinoma (13), non-small cell lung cancer (14), breast cancer (15), gastric cancer (16), and renal cell carcinoma (17). Additionally, in some hematological tumors, such as nonHodgkin lymphoma and multiple myeloma, NLR has been recognized to play an important role in predicting response and survival rates in patients (18-20). There are little literatures reported about NLR applied to predict the prognosis of AML. Mushtaq et al made a research about the connection between NLR and OS in patients with relapsed/ refractory AML (21). However, the relation of NLR at diagnose with the prognosis of de-novo AML patients remains unknown. Moreover, we considered that M3 is different from other types of leukemia. For patients with non-M3 leukemia, we adopt routine chemotherapy, but for patients with M3, we use different treatments, using alltrans retinoic acid and anthracycline. So we choose non-M3 de novo AML patients for our study.

In this study, we investigate the prognostic value of pretreatment NLR and the utility of NLR to predict the response to chemotherapy in a cohort of 181 newly diagnosed non-M3 AML patients. We found that patients with pretreatment NLR below 2.0 possessed a substantially better prognosis than those with higher NLR. Additionally, when patients achieved complete remission (CR), median NLR was dramatically increased in patients with low NLR group, but had no statistical change in patients with high NLR group.

We present the following article in accordance with the REMARK reporting checklist (available at http://dx.doi. org/10.21037/tcr-20-2179).

\section{Methods}

\section{Patients}

From June 2007 to December 2015, a total of 181 newly diagnosed patients with de novo non-M3 AML at the First Affiliated Hospital of Wenzhou Medical University were enrolled as previously described (3). All patients (98 males and 83 females, median age 40 years, range, 14-60) were diagnosed and classified according to WHO 2008 classification criteria for AML (22) and were required to receive induction chemotherapy for at least one course with intact follow-up records. The study was conducted in accordance with the Declaration of Helsinki (as revised in 2013). The study was approved by the Institutional Ethics Committee of the First Affiliated Hospital of Wenzhou Medical University (No. 2016-081) and informed consent was taken from all the patients.

Before the patients undergoes chemotherapy, the patients were evaluated NLR, which were calculated by the ratio of the number of neutrophils to lymphocytes in the peripheral blood (PB). In this way, the patients were categorized into two groups by defining NLR $=2.0$ as the cut-off point.

After initial diagnosis, a majority of patients received IA (idarubicin $8-10 \mathrm{mg} / \mathrm{m}^{2}$ per day on days $1-3$ and cytarabine $100 \mathrm{mg} / \mathrm{m}^{2}$ per day on days $\left.1-7\right), 4$ patients received DA (daunorubicin $45-60 \mathrm{mg} / \mathrm{m}^{2}$ per day on days $1-3$ and cytarabine $100 \mathrm{mg} / \mathrm{m}^{2}$ per day on days $\left.1-7\right)$, and 9 patients 
received HA (homoharringtonine $4-6 \mathrm{mg} / \mathrm{m}^{2}$ per day on days $1-7$ and cytarabine $100 \mathrm{mg} / \mathrm{m}^{2}$ per day on days $1-7$ ). A total of 100 patients achieved hematological CR after the first course of induction chemotherapy. And there were 24 patients did not achieve CR after two cycles of induction chemotherapy. Patients subsequently received consolidation with high doses of cytarabine [Ara-C 1.5$3.0 \mathrm{~g} / \mathrm{m}^{2}$ q12h d1-3 for 4 courses or until they underwent allogeneic hematopoietic stem cell transplantation (HSCT)]. Additionally, 53 patients underwent high-dose chemotherapy and allogeneic HSCT as a post-remission therapy.

\section{Cytogenetic analysis}

The vast majority of patients had cytogenetic analysis at the initial time of diagnosis by R- and/or G-banding techniques and classification according to the International System for Human Cytogenetic Nomenclature. Risk status was classified to favorable, intermediate and unfavorable risk according to NCCN guidelines (5). In general, favorable risk mainly included patients with $\mathrm{t}(8 ; 21)$, inv(16) or $\mathrm{t}(16 ; 16)$; unfavorable risk included patients with a complex karyotype ( $\geq 3$ clonal chromosomal abnormalities), monosomal karyotype, abnormalities of chromosome 5 and/ or $7,11 \mathrm{q} 23$ - non $\mathrm{t}(9 ; 11), \mathrm{t}(6 ; 9) ; \mathrm{t}(9 ; 22)$, inv(3) or $\mathrm{t}(3 ; 3)$; and intermediate risk referred to patients with other findings including normal cytogenetics, +8 alone, $\mathrm{t}(9 ; 11)$ and other non-defined.

\section{Statistical analysis}

Overall survival (OS) was calculated from the date of diagnosis until the date of last contact or death. Diseasefree survival (DFS) was calculated from the documented date of CR until date of relapse or death from any cause. Relapse was defined by recurrence of $>5 \%$ blasts in the BM unrelated to recovery or by the presence of extramedullary disease. OS and DFS rates were calculated using the Kaplan-Meier methods, and the curves were compared using the log-rank test. Kruska-Wallis $\mathrm{H}$ test was used to compare continuous variables and Chi-square test or Fisher's exact test was used to compare categorical variables. Variables of with $\mathrm{P}<0.05$ in the univariate Cox regression analysis were selected for multivariate analysis. All statistical tests were two-sided and a $\mathrm{P}$ value less than 0.05 was considered statistically significant. Statistical analyses were carried out using SPSS software (ver. 24.0).

\section{Results}

\section{Patient characteristics of AML patients}

The baseline characteristics of 181 patients were summarized in Table 1. Median WBC count was $14.6 \times 10^{9}$ / L (range: $\left.0.38 \times 10^{9}-464 \times 10^{9} / \mathrm{L}\right)$. The median percentages of blasts in $\mathrm{PB}$ and $\mathrm{BM}$ were $59 \%$ (range: $0-98 \%$ ) and $66.0 \%$ (range: 9.0-98.8\%), respectively. According to the WHO classification, 23 (12.7\%) were AML with $\mathrm{t}(8 ; 21)(\mathrm{q} 22 ; \mathrm{q} 22)$; RUNX1-RUNX1T1, 5 (2.8\%) were AML with inv (16) (p13.1;q22) or $\mathrm{t}(16 ; 16)(\mathrm{p} 13.1 ; \mathrm{q} 22)$; CBFB-MYH11, 23 (12.7\%) were AML with maturation, 69 (38.1\%) were acute myelomonocytic leukemia, $50(27.6 \%)$ were acute monoblastic/monocytic leukemia, 7 (3.9\%) were acute erythroid leukemia, $1(0.6 \%)$ were acute megakaryoblastic leukemia, and the remaining $3(1.7 \%)$ patients were unclassified. Among these patients, 178 patients had available cytogenetic analysis at diagnosis. A total of 17 , 144 and 17 patients showed favorable, intermediate, and unfavorable karyotype, respectively. The estimated 5-year OS was $41.9 \%$ [ $95 \%$ confidence interval (CI): $33.6-50.2 \%$ ] and the estimated 5-year DFS was $40.3 \%$ (95\% CI: 31.8 $48.8 \%)$. At the time of analysis, the median follow-up was 28.0 months (range, $0-134$ months) and 90 of 181 (49.7\%) patients died.

The median NLR for all patients at diagnosis was 0.4878 , with $86.74 \%$ and $13.26 \%$ patients showed low NLR $(<2.0)$ and high NLR $(\geq 2.0)$, respectively. Patients between low NLR and high NLR showed a significant difference in blasts in BM, and those who had high NLR tended to have little blasts in $\mathrm{BM}(\mathrm{P}=0.005)$. Absolute neutrophil count (ANC) tended to have a relationship with NLR. Higher ANC was related with higher NLR $(\mathrm{P}<0.001)$. Patient characteristics grouped according to pretreatment NLR were summarized in Table 1.

\section{NLR correlates with prognosis and treatment outcome in patients with no-M3 AML}

With a median follow-up of 28 months (range, 0 134 months), there was a significant difference in OS between low NLR and high NLR $(\mathrm{P}=0.028)$ (Figure 1A). Kaplan-Meier analysis also showed a significant difference in DFS between the two groups $(\mathrm{P}=0.036)$ (Figure 1B). The univariate Cox regression analysis in Table 2 showed the following clinical parameters were significantly associated with OS: age (years), ANC (as continues variable), absolute lymphocyte count (ALC) (as continues variable) and NLR 
Table 1 Baseline patient characteristics

\begin{tabular}{|c|c|c|c|c|}
\hline Characteristics & All patients $(n=181)$ & Low NLR $(<2.0)(n=157)$ & High NLR $(\geq 2.0)(n=24)$ & $P$ value \\
\hline Male/female & $98 / 83$ & $83 / 74$ & $15 / 9$ & 0.378 \\
\hline Median WBC count (range), $\times 10^{9} / \mathrm{L}$ & $14.6(0.38-464.00)$ & $14.09(0.38-464.00)$ & $39.22(3.35-254.20)$ & 0.006 \\
\hline Median hemoglobin (range), g/L & $74.00(34.0-153.00)$ & $77.00(34.0-153.00)$ & $67.50(45.00-115.00)$ & 0.089 \\
\hline Median platelets (range), $\times 10^{9} / \mathrm{L}$ & $36.0(2.0-376.0)$ & $36.0(2.0-280.0)$ & $40.5(5.0-376.0)$ & 0.537 \\
\hline Median blasts in BM (range), \% & $66.0(9.0-98.8)$ & $70.4(9.0-98.8)$ & $50.0(15.2-94.5)$ & 0.005 \\
\hline Median ANC (range), $\times 10^{9} / \mathrm{L}$ & $1.2824(0.0072-31.8300)$ & $0.9816(0.0072-31.8300)$ & $8.1699(1.8564-30.3730)$ & $<0.001$ \\
\hline Median ALC (range), $\times 10^{9} / \mathrm{L}$ & $2.7440(0.2544-43.6656)$ & $2.7828(0.2544-43.6656)$ & $2.2188(0.4395-8.6780)$ & 0.279 \\
\hline Median NLR (range) & $0.4878(0.0197-10.4000)$ & $0.3333(0.0197-1.8571)$ & $3.4300(2.0000-10.4000)$ & $<0.001$ \\
\hline$\geq 38.5$ & $54(29.8)$ & $47(29.9)$ & $7(29.2)$ & \\
\hline \multicolumn{5}{|l|}{ Subtypes, n (\%) } \\
\hline AML with $\mathrm{t}(8 ; 21)$ (q22;q22); RUNX1-RUNX1T1 & $23(12.7)$ & $18(11.5)$ & $5(20.8)$ & 0.340 \\
\hline $\begin{array}{l}\text { AML with inv[16] (p13.1;q22) or t(16;16) } \\
\text { (p13.1;q22); CBFB-MYH11 }\end{array}$ & $5(2.8)$ & $5(3.2)$ & 0 & 1 \\
\hline AML with mutated NPM1 & 0 & 0 & 0 & 1 \\
\hline AML with mutated CEBPA & $3(1.7)$ & $3(1.9)$ & 0 & 1 \\
\hline AML with maturation & $20(11.0)$ & $16(10.2)$ & $4(16.7)$ & 0.553 \\
\hline Favorable & $17(9.4)$ & $14(8.9)$ & $3(12.5)$ & \\
\hline Intermediate & $144(79.6)$ & $126(80.3)$ & $18(75.0)$ & \\
\hline Unfavorable & $17(9.4)$ & $15(9.6)$ & $2(8.3)$ & \\
\hline Missing & $3(1.7)$ & $2(1.3)$ & $1(4.2)$ & \\
\hline Induction chemotherapy, n (\%) & & & & 0.803 \\
\hline IA & $168(92.8)$ & $146(93.0)$ & $22(91.7)$ & \\
\hline DA & $4(2.2)$ & $3(1.9)$ & $1(4.2)$ & \\
\hline $\mathrm{HA}$ & $9(5.0)$ & $8(5.1)$ & $1(4.2)$ & \\
\hline $\mathrm{CR}^{\mathrm{b}}, \mathrm{n}(\%)$ & $157(86.7)$ & $138(87.9)$ & $19(79.2)$ & 0.394 \\
\hline Relapse, n (\%) & $72(45.9)$ & $60(43.5)$ & $12(63.2)$ & 0.107 \\
\hline No. of patients who underwent HSCT, n (\%) & $53(29.3)$ & $49(31.2)$ & $4(16.7)$ & 0.145 \\
\hline
\end{tabular}

${ }^{a}$, comparison of the two cytogenetic subgroups (favorable versus other); ${ }^{b}$, achieved complete remission (CR) after one course of induction therapy. WBC, white blood cell; PB, peripheral blood; BM, bone marrow; ANC, absolute neutrophil count; ALC, absolute lymphocyte count; HSCT, hematopoietic stem cell transplant. 

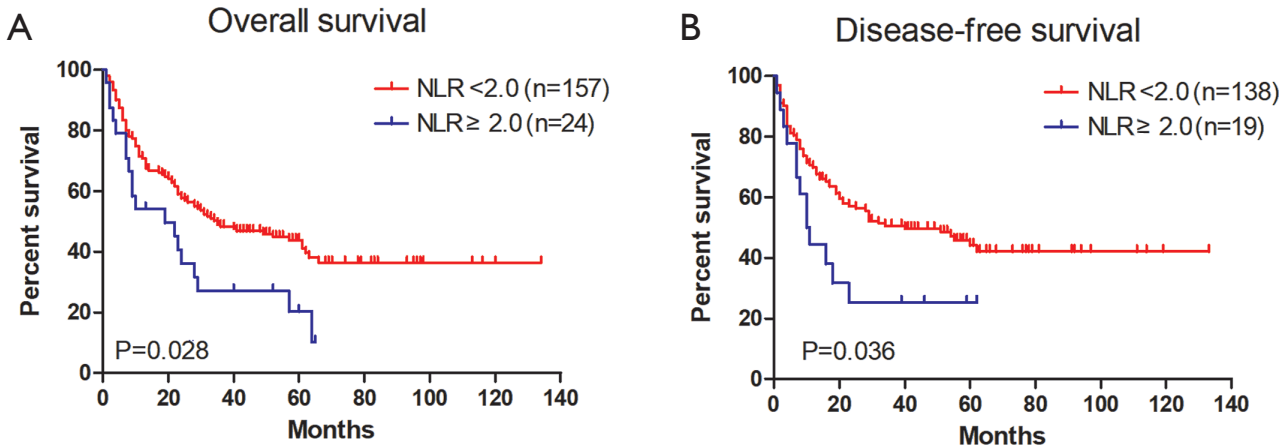

Figure 1 Survival outcomes of patients with acute myeloid leukemia grouped according to pretreatment NLR. (A) Overall survival after diagnosis was compared between AML patients with low NLR and high NLR. (B) Disease-free survival after complete remission was compared between AML patients with low NLR and high NLR. AML, acute myeloid leukemia; NLR, neutrophil-to-lymphocyte.

Table 2 Univariate analyses of clinical factors for OS and DFS

\begin{tabular}{|c|c|c|c|c|c|c|}
\hline Characteristics & \multicolumn{3}{|c|}{ Os } & \multicolumn{3}{|c|}{ DFS } \\
\hline Age (years) & 1.026 & $1.011-1.042$ & 0.001 & 1.022 & $1.007-1.037$ & 0.005 \\
\hline Gender & 0.830 & $0.573-1.201$ & 0.323 & 0.874 & $0.604-1.264$ & 0.474 \\
\hline $\log (\mathrm{WBC})$ & 1.486 & $1.095-2.016$ & 0.011 & 1.451 & $1.073-1.963$ & 0.016 \\
\hline PLT ( $\times 10^{9} / L,<100$ vs. $\left.\geq 100\right)$ & 0.874 & $0.544-1.405$ & 0.578 & 0.784 & $0.488-1.260$ & 0.315 \\
\hline $\operatorname{NLR}(<2.0$ vs. $\geq 2.0)$ & 0.591 & $0.360-0.970$ & 0.038 & 0.555 & $0.338-0.912$ & 0.020 \\
\hline ANC $\left(\times 10^{9} / L\right.$, continues variable) & 1.054 & $1.023-1.086$ & 0.001 & 1.046 & $1.016-1.076$ & 0.002 \\
\hline ALC $\left(\times 10^{9} / \mathrm{L}\right.$, continues variable $)$ & 1.066 & $1.031-1.102$ & $<0.001$ & 1.048 & $1.016-1.081$ & 0.003 \\
\hline
\end{tabular}

WBC, white blood cell; HB, hemoglobin; PLT, platelet; PB, peripheral blood; BM, bone marrow; ANC, absolute neutrophil count; ALC, absolute lymphocyte count; NLR, neutrophil to lymphocyte ratio. 95\% Cl, 95\% confidence interval.

( $\mathrm{P}=0.001, \mathrm{P}=0.001, \mathrm{P}<0.001$ and $\mathrm{P}=0.038$, respectively).

Multivariate analyses adjusting for age, ANC, ALC and NLR, showed that age or ALC was markedly and independently associated with $\mathrm{OS}(\mathrm{P}=0.001$ and $\mathrm{P}=0.006$, respectively) and DFS ( $\mathrm{P}=0.004$ and $\mathrm{P}=0.050$, respectively), and the other two failed to reach the statistical difference (Table 3). No statistical difference was observed in NLR between de novo AML patients who achieved CR and did not achieve $\mathrm{CR}(\mathrm{P}>0.05$, Figure $2 A)$. We further investigated the relationship of NLR and response to therapy and found that NLR was considerably increased when these patients achieved CR regardless of the type of treatments received or only received IA therapy (both $\mathrm{P}<0.01$, Figure $2 B, C$ ).
Specifically in patients with low NLR, NLR was significantly increased when these patients achieved CR regardless of treatments received or only received IA therapy (both $\mathrm{P}<0.01$, Figure $2 D, E)$. However, in patients with high initial NLR, there had no statistical change when these patients achieved CR through only receiving IA therapy or including other types of treatment (both $\mathrm{P}>0.05$, Figure $2 F, G$ ). In addition, there was no correlation between NLR and the risk stratification of cytogenetics in all patients (Figure $3 \mathrm{~A}$ ). When these patients achieved CR, NLR was significantly up-regulated in all three groups, and patients with favorable risk were highest among three groups (Figure 3B). Taken together, these data suggested that initial NLR can as 
Table 3 Multivariate analysis of clinical factors for OS

\begin{tabular}{|c|c|c|c|c|c|c|}
\hline Characteristics & \multicolumn{3}{|c|}{ OS } & \multicolumn{3}{|c|}{ DFS } \\
\hline Age (years) & 1.026 & $1.011-1.042$ & 0.001 & 1.022 & $1.007-1.037$ & 0.004 \\
\hline $\log (\mathrm{WBC})$ & 1.040 & $0.716-1.511$ & 0.837 & 1.129 & $0.776-1.643$ & 0.526 \\
\hline $\operatorname{NLR}(<2.0$ vs. $\geq 2.0)$ & 0.567 & $0.291-1.104$ & 0.095 & 0.516 & $0.261-1.022$ & 0.058 \\
\hline ALC $\left(\times 10^{9} / \mathrm{L}\right.$, continues variable $)$ & 1.061 & $1.017-1.107$ & 0.006 & 1.041 & $1.000-1.085$ & 0.050 \\
\hline
\end{tabular}

WBC, white blood cell; NLR, neutrophil to lymphocyte ratio; ANC, absolute neutrophil count; ALC, absolute lymphocyte count. 95\% CI, $95 \%$ confidence interval.

an independent prognostic biomarker and NLR can reflect the response to treatment in patients with non-M3 AML.

\section{Prognostic impact of NLR for patients with AML with myeloblasts over $50 \%$ in BM}

Because the multivariate Cox analysis showed that NLR had a slight but not statistically significant correlation with OS and DFS in all patients, we regrouped the patients with higher myeloblasts in $\mathrm{BM}(>50 \%)$. The patient characteristics regrouped were shown in Table 4. A significant difference was observed in ANC, NLC between low NLR group and high NLR group $(\mathrm{P}<0.001)$. KaplanMeier analysis showed that higher NLR was associated with short OS and DFS, when compared with lower NLR in patients with myeloblasts over $50 \%$ in $\mathrm{BM}(\mathrm{P}<0.001$ and $\mathrm{P}=0.004$, respectively) (Figure $4 A, B$ ).

As presented in Table 5, the univariate analysis included $\log (\mathrm{WBC})$, NLR, ANC, ALC as parameters were significantly associated with $\mathrm{OS}(\mathrm{P}=0.043, \mathrm{P}<0.001$, $\mathrm{P}<0.001$, and $\mathrm{P}<0.001$, respectively). The following clinical parameters were significantly associated with DFS: NLR, ANC, ALC $(\mathrm{P}=0.001, \mathrm{P}=0.001$, and $\mathrm{P}<0.001$, respectively). In the multivariate analysis shown in Table 5, both NLR and ALC showed a significant association with DFS and OS, respectively. Additionally, we also assessed the relationship of NLR and response to treatment in these cases with higher myeloblasts in BM, similar results were also found (Figure 5).

\section{Discussion}

A variety of prior studies have reported that higher NLR is correlated with a worse survival outcome, in both solid tumors and hematopoietic tumors (23-26). In the present study, we identified NLR $=2.0$ as the cut-off point according to two previous studies. Jung et al. (27) reported that low NLR $(<2.0)$ group showed significantly better response rates to induction chemotherapy compared with the high NLR $(\geq 2.0)$ group and had a better 3 -year OS in patients with primary central nervous system lymphoma. And $\mathrm{Li}$ et al. (28) reported that high NLR $(\geq 2.0)$ group experienced shorter OS and PFS compared with low NLR $(<2.0)$ group in patients with multiple myeloma. Thus we also divided patients into two subgroups, as low NLR $(<2.0)$ and high NLR $(\geq 2.0)$ group. To our knowledge, this is the first study demonstrated pretreatment NLR as an indicator to predict prognosis in AML patients. Shorter OS and DFS were observed in those with high NLR. Moreover, we also found that NLR was significantly increased when patients achieved CR after induction chemotherapy.

In the tumor microenvironment, NLR reflects the immune responses in patients. It has been reported that persistent chronic inflammation could be a triggered factor to tumorigenesis (29-31). Neutrophils are not only the defenses in anti-inflammation but also secrete cytokines which further promote cancer development, such as interleukin (IL)-2, IL-10, and tumor necrosis factor $\alpha$ (32). In contrast, lymphocytes play an important role in defensing against cancer cells $(33,34)$. In our study, a statistical difference was also observed in pretreatment NLR in all patients including those cases with myeloblasts over $50 \%$ in BM, which may be caused by difference in the inflammatory level and the degree of immune response. So we preferred to regard NLR as a potential marker for inflammatory burden and tumor load. In AML patients, high NLR is often accompanied by a lower ALC, which may indicate a decrease in antitumor response in vivo 
A

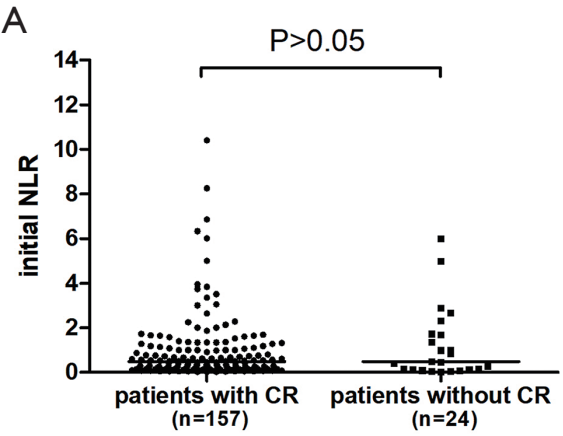

C

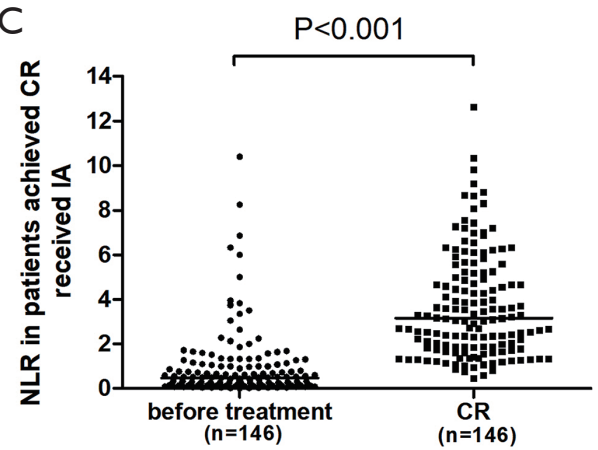

$\mathrm{E}$

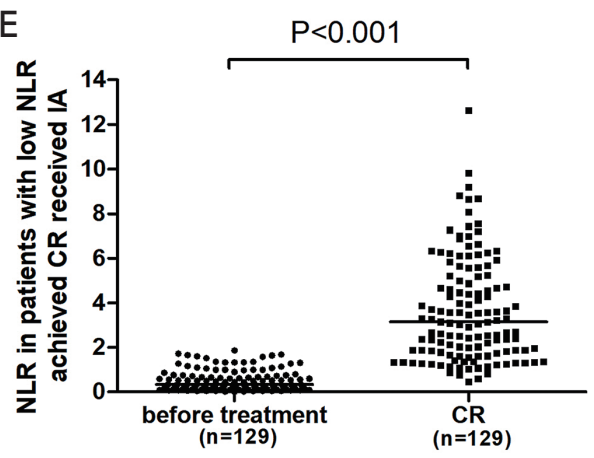

G

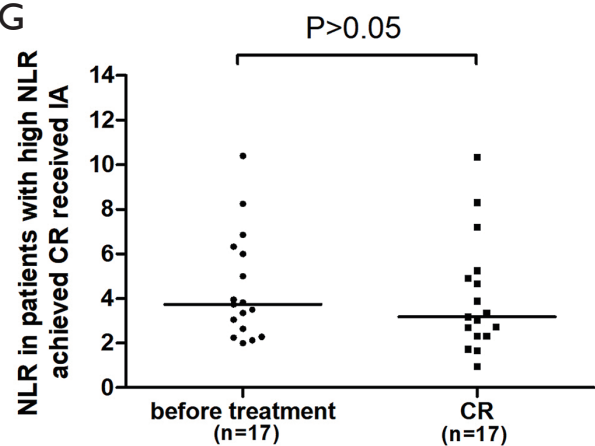

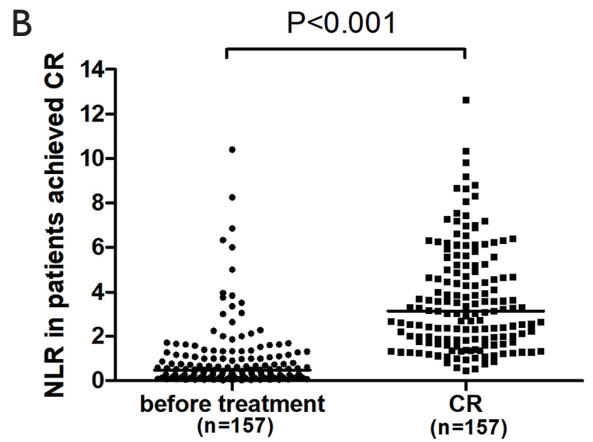
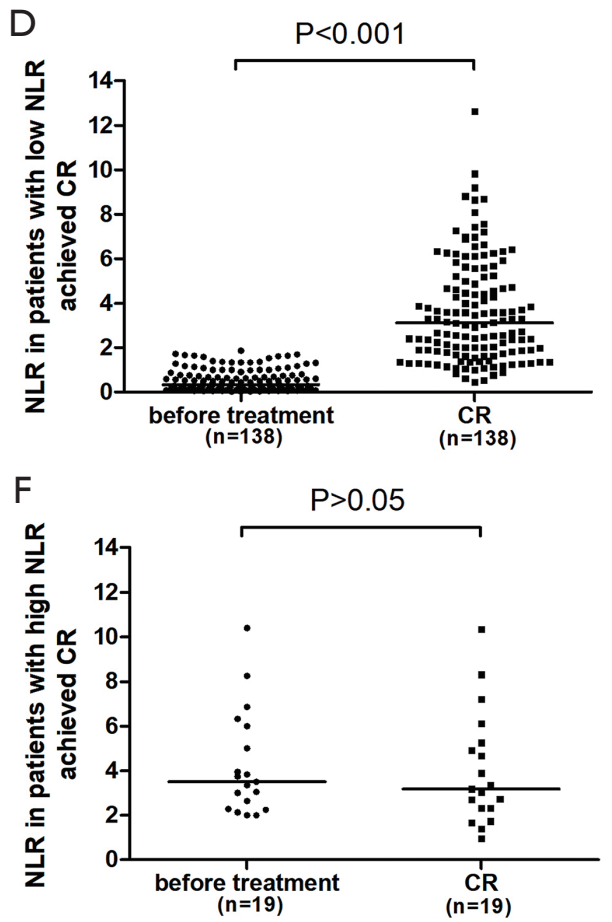

Figure 2 NLR is upregulated in patients with non-M3 AML when these patients achieve complete remission. (A) No difference was observed in initial NLR in patients who achieve CR and did not achieve CR. (B,C) NLR was considerably increased when patients achieved CR regardless of treatments received or only receiving IA therapy. (D,E) NLR was significantly increased when patients with low initial NLR achieved CR regardless of treatments received or only receiving IA therapy. (F,G) No statistical change was observed in NLR when patients with high initial NLR achieved CR regardless of treatments received or only receiving IA therapy. Differences between medians of two groups were determined using Kruskal-Wallis H test. AML, acute myeloid leukemia; NLR, neutrophil-to-lymphocyte; CR, complete remission. 

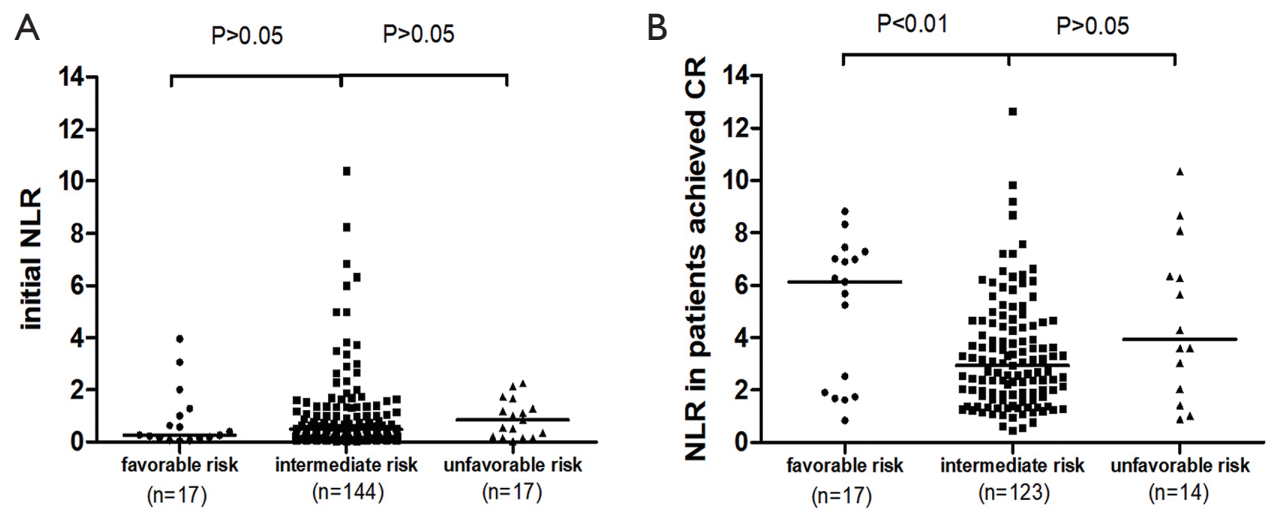

Figure 3 NLR has a slight link to cytogenetic classification in non-M3 AML patients. (A) There was no correlation of initial NLR and risk stratification in patients with non-M3 AML. (B) NLR in patients with favorable risk was highest among three groups when these patients achieved complete remission. Mann-Whitney $U$ test was used to test difference among medians of NLR in three groups according to cytogenetic classification.

Table 4 Baseline for patients with myeloblasts over 50\% in BM at diagnose

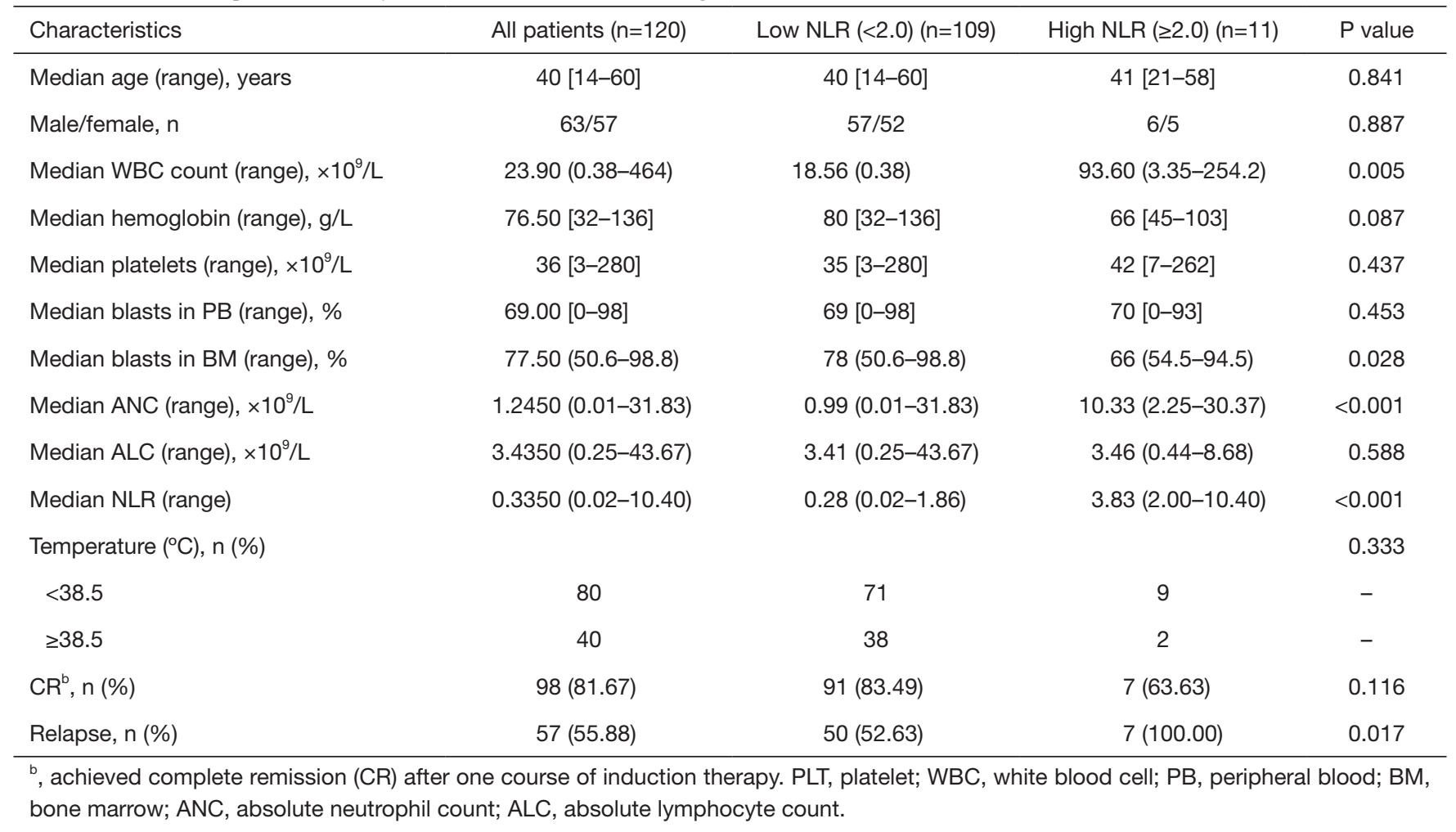

reported previously (35), which finally results in a worse survival outcome. Additionally, initial ALC also appeared as an independent predictor of OS in these patients, similar to a previous report (35).

Although our study has many advantages over previous studies, it also exists limitations. First of all, selection bias was unavoidable and difficult to be well balanced in our study. For examples, proportions of AML with maturation and acute myelomonocytic leukemia subtypes were $12.7 \%$ and $38.1 \%$ in our cohort, which slightly differed from 

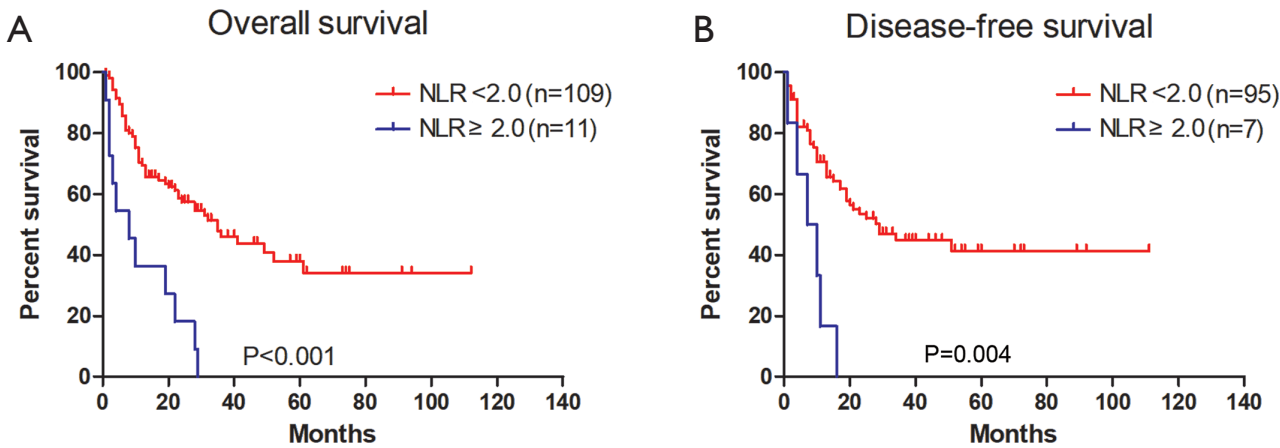

Figure 4 Survival outcomes of AML patients with myeloblasts over 50\% in BM according to pretreatment NLR. (A) Overall survival after diagnosis was compared between patients with myeloblasts over 50\% in BM with low NLR and high NLR. (B) Disease-free survival after complete remission was compared between patients with myeloblasts over $50 \%$ in BM with low NLR and high NLR.

Table 5 Univariate, multivariate analyses of patients with myeloblasts over $50 \%$ in BM for OS and DFS

\begin{tabular}{|c|c|c|c|c|c|c|}
\hline Characteristics & \multicolumn{3}{|c|}{ Os } & \multicolumn{3}{|c|}{ DFS } \\
\hline \multicolumn{7}{|l|}{ Univariate analyses } \\
\hline $\log (\mathrm{WBC})$ & 1.524 & $1.014-2.290$ & 0.043 & 1.165 & $0.780-1.739$ & 0.455 \\
\hline $\mathrm{HB}$ (g/L, <100 vs. $\geq 100)$ & 0.482 & $0.428-1.429$ & 0.424 & 1.019 & $0.577-1.802$ & 0.948 \\
\hline ANC $\left(\times 10^{9} / \mathrm{L}\right.$, continues variable $)$ & 1.072 & $1.037-1.107$ & $<0.001$ & 1.060 & $1.023-1.099$ & 0.001 \\
\hline ALC $\left(\times 10^{9} / \mathrm{L}\right.$, continues variable $)$ & 1.078 & $1.042-1.116$ & $<0.001$ & 1.068 & $1.031-1.106$ & $<0.001$ \\
\hline Blasts in PB (\%, $\leq 20$ vs. $>20)$ & 1.230 & $0.650-2.327$ & 0.524 & 1.012 & $0.544-1.880$ & 0.971 \\
\hline \multicolumn{7}{|l|}{ Multivariate analyses } \\
\hline ALC $\left(\times 10^{9} / \mathrm{L}\right.$, continues variable) & 1.083 & $1.042-1.127$ & $<0.001$ & 1.076 & $1.034-1.120$ & $<0.001$ \\
\hline
\end{tabular}

HB, hemoglobin; PLT, platelet; PB, peripheral blood; BM, bone marrow; ANC, absolute neutrophil count; ALC, absolute lymphocyte count; NLR, neutrophil to lymphocyte ratio. $95 \% \mathrm{Cl}, 95 \%$ confidence interval.

the previously published population-based studies in western countries $(36,37)$. Secondly, a portion of patients only completed BM morphologic and immunologic and cytogenetic analysis, but no molecular biologic analysis due to high medical costs. Pretreatment NLR as a potential prognostic indicator could not be well-validated in the multivariate context including genetic information in this study. Thirdly, a limitation in our study was the bias caused by HSCT. We did not give an additional explanation as no statistical significance was observed in the patients who underwent HSCT or not. Lastly, both neutrophils and lymphocytes are closely related to tumor immunity, further studies need to be performed to verify the detailed mechanisms.

\section{Conclusions}

We demonstrated the relationship between NLR at diagnosis and prognosis in patients with AML excluding M3 and found that NLR could be used as an independent indictor to predict the prognosis of patients with AML excluding $\mathrm{M} 3$, especially in those cases with myeloblasts 
A

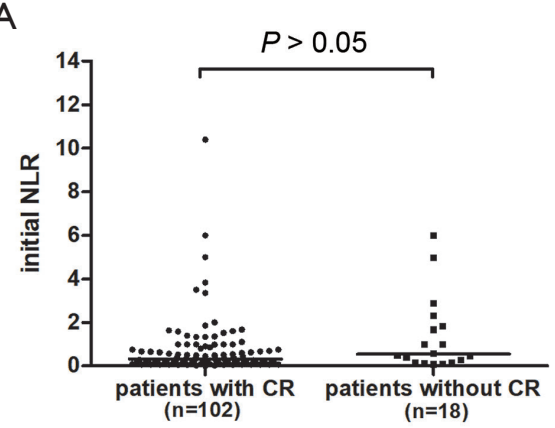

C

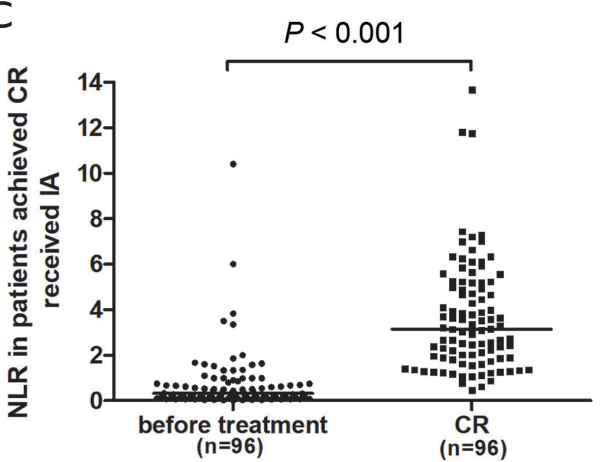

E

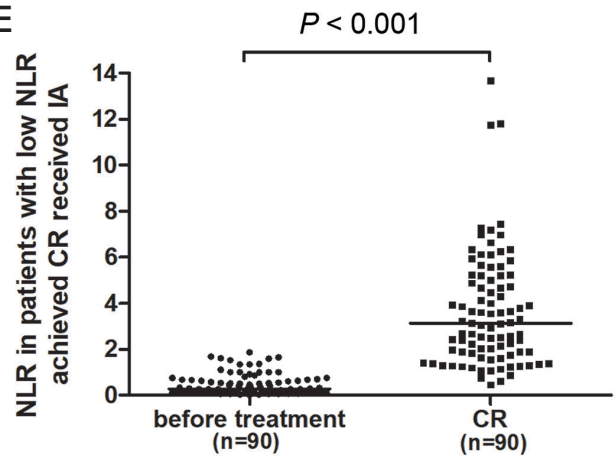

G

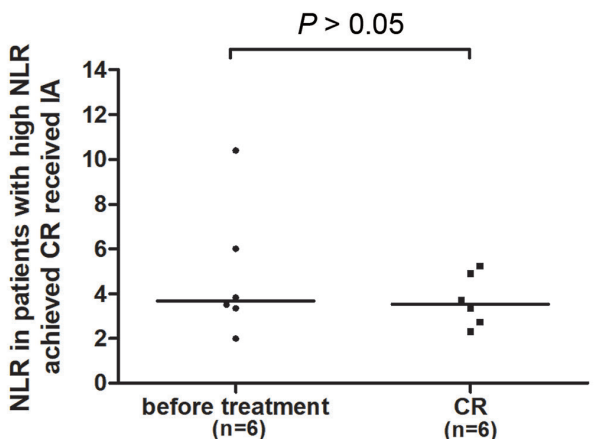

B

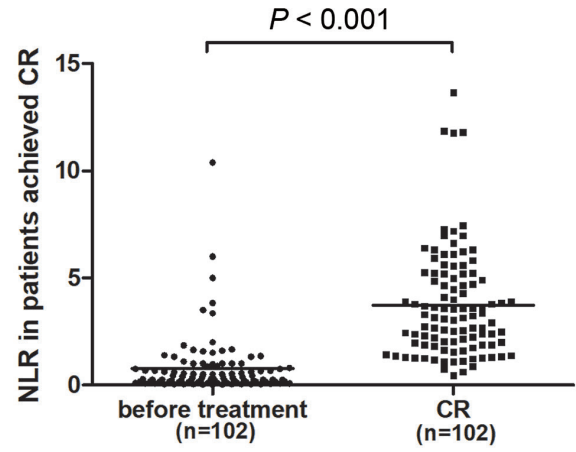

D

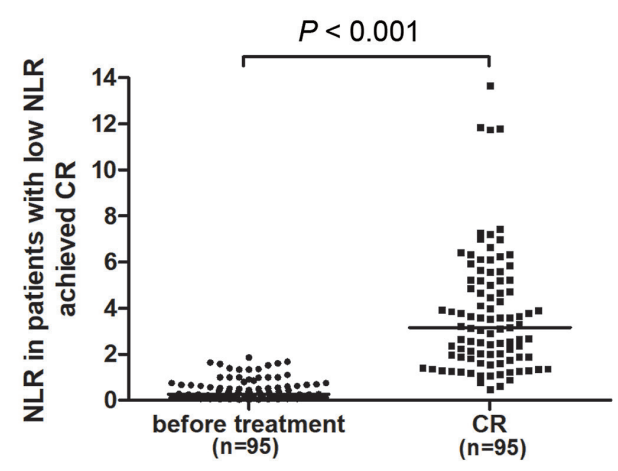

$\mathrm{F}$

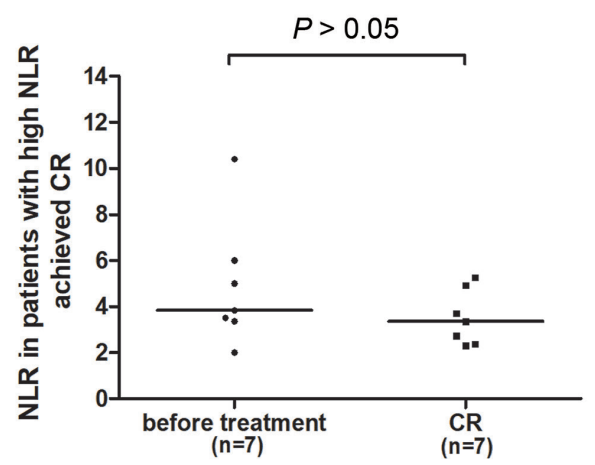

Figure 5 NLR is increased in AML patients with myeloblasts over 50\% in BM when these patients achieve complete remission. (A) There was no difference in initial NLR in patients who achieve complete remission (CR) and did not achieve CR. (B,C) NLR was significantly increased when patients achieved CR regardless of treatments received or only receiving IA therapy. (D,E) NLR was dramatically increased when patients with low initial NLR achieved CR regardless of treatments received or only receiving IA therapy. (F,G) There was no statistical in NLR when patients with high initial NLR achieved CR regardless of treatments received or only receiving IA therapy. KruskalWallis $\mathrm{H}$ test was used to determine differences between medians of two groups. 
over $50 \%$ in BM. NLR can also reflect the response to therapy after induction chemotherapy in AML patients.

\section{Acknowledgments}

Funding: This work was supported by Zhejiang Provincial Natural Science Foundation of China (No. LY20H080005, LY17H080005, LY17H080006), and the grant of Wenzhou Municipal Science and Technology Bureau (No. Y20150006, Y20150031).

\section{Footnote}

Reporting Checklist: The authors have completed the REMARK reporting checklist. Available at http://dx.doi. org/10.21037/tcr-20-2179

Data Sharing Statement: Available at http://dx.doi. org/10.21037/tcr-20-2179

Conflicts of Interest: All authors have completed the ICMJE uniform disclosure form (available at http://dx.doi. org/10.21037/tcr-20-2179). The authors have no conflicts of interest to declare.

Ethical Statement: The authors are accountable for all aspects of the work in ensuring that questions related to the accuracy or integrity of any part of the work are appropriately investigated and resolved. The study was conducted in accordance with the Declaration of Helsinki (as revised in 2013). The study was approved by the Institutional Ethics Committee of the First Affiliated Hospital of Wenzhou Medical University (No. 2016-081) and informed consent was taken from all the patients.

Open Access Statement: This is an Open Access article distributed in accordance with the Creative Commons Attribution-NonCommercial-NoDerivs 4.0 International License (CC BY-NC-ND 4.0), which permits the noncommercial replication and distribution of the article with the strict proviso that no changes or edits are made and the original work is properly cited (including links to both the formal publication through the relevant DOI and the license). See: https://creativecommons.org/licenses/by-nc-nd/4.0/.

\section{References}

1. Amatangelo MD, Quek L, Shih A, et al. Enasidenib induces acute myeloid leukemia cell differentiation to promote clinical response. Blood 2017;130:732-41.

2. Dohner H, Weisdorf DJ, Bloomfield CD. Acute Myeloid Leukemia. N Engl J Med 2015;373:1136-52.

3. Zhang Q, Dai K, Bi L, et al. Pretreatment platelet count predicts survival outcome of patients with de novo non-M3 acute myeloid leukemia. PeerJ 2017;5:e4139.

4. Dai K, Zhang Q, Li Y, et al. Plasma fibrinogen levels correlate with prognosis and treatment outcome in patients with non-M3 acute myeloid leukemia. Leuk Lymphoma 2019;60:1503-11.

5. O'Donnell MR, Tallman MS, Abboud CN, et al. Acute Myeloid Leukemia, Version 3.2017, NCCN Clinical Practice Guidelines in Oncology. J Natl Compr Canc Netw 2017;15:926-57.

6. Carey A, Edwards DKt, Eide CA, et al. Identification of Interleukin-1 by Functional Screening as a Key Mediator of Cellular Expansion and Disease Progression in Acute Myeloid Leukemia. Cell Rep 2017;18:3204-18.

7. Balkwill F, Mantovani A. Inflammation and cancer: back to Virchow? Lancet 2001;357:539-45.

8. Han Y, Ye A, Bi L, et al. Th17 cells and interleukin-17 increase with poor prognosis in patients with acute myeloid leukemia. Cancer Sci 2014;105:933-42.

9. Pietras EM. Inflammation: a key regulator of hematopoietic stem cell fate in health and disease. Blood 2017;130:1693-8.

10. Hemmati S, Haque T, Gritsman K. Inflammatory Signaling Pathways in Preleukemic and Leukemic Stem Cells. Front Oncol 2017;7:265.

11. Mantovani A, Allavena P, Sica A, et al. Cancer-related inflammation. Nature 2008;454:436-44.

12. Wu Y, Zhao Q, Peng C, et al. Neutrophils promote motility of cancer cells via a hyaluronan-mediated TLR4/ PI3K activation loop. J Pathol 2011;225:438-47.

13. Okamura Y, Sugiura T, Ito T, et al. Neutrophil to lymphocyte ratio as an indicator of the malignant behaviour of hepatocellular carcinoma. Br J Surg 2016;103:891-8.

14. Gu XB, Tian T, Tian XJ, et al. Prognostic significance of neutrophil-to-lymphocyte ratio in non-small cell lung cancer: a meta-analysis. Sci Rep 2015;5:12493.

15. Chen J, Deng Q, Pan Y, et al. Prognostic value of neutrophil-to-lymphocyte ratio in breast cancer. FEBS Open Bio 2015;5:502-7.

16. Sun J, Chen X, Gao P, et al. Can the Neutrophil to Lymphocyte Ratio Be Used to Determine Gastric Cancer Treatment Outcomes? A Systematic Review and Meta- 
Analysis. Dis Markers 2016;2016:7862469.

17. Hu K, Lou L, Ye J, et al. Prognostic role of the neutrophillymphocyte ratio in renal cell carcinoma: a meta-analysis. BMJ Open 2015;5:e006404.

18. Wang J, Zhou X, Liu Y, et al. Prognostic significance of neutrophil-to-lymphocyte ratio in diffuse large B-cell lymphoma: A meta-analysis. PLoS One 2017;12:e0176008.

19. Onec B, Okutan H, Albayrak M, et al. The Predictive Role of the Neutrophil/Lymphocyte Ratio in Survival with Multiple Myeloma: A Single Center Experience. J Clin Lab Anal 2017;31:e22032.

20. Stefaniuk P, Szymczyk A, Podhorecka M. The Neutrophil to Lymphocyte and Lymphocyte to Monocyte Ratios as New Prognostic Factors in Hematological Malignancies A Narrative Review. Cancer Manag Res 2020;12:2961-77.

21. Mushtaq MU, Chaudhary SG, Murthy GSG, et al. Prognostic Significance of Neutrophil-to-Lymphocyte Ratio in Relapsed/Refractory Acute Myeloid Leukemia. Blood 2018;132:5246.

22. Vardiman JW, Thiele J, Arber DA, et al. The 2008 revision of the World Health Organization (WHO) classification of myeloid neoplasms and acute leukemia: rationale and important changes. Blood 2009;114:937-51.

23. Song Y, Yang Y, Gao P, et al. The preoperative neutrophil to lymphocyte ratio is a superior indicator of prognosis compared with other inflammatory biomarkers in resectable colorectal cancer. BMC Cancer 2017;17:744.

24. Wang J, Zhou M, Xu JY, et al. Prognostic role of pretreatment neutrophil-lymphocyte ratio in patients with diffuse large B-cell lymphoma treated with RCHOP. Medicine (Baltimore) 2016;95:e4893.

25. Zhou B, Deng J, Chen L, et al. Preoperative neutrophilto-lymphocyte ratio and tumor-related factors to predict lymph node metastasis in nonfunctioning pancreatic neuroendocrine tumors. Sci Rep 2017;7:17506.

26. Azab B, Bhatt VR, Phookan J, et al. Usefulness of the neutrophil-to-lymphocyte ratio in predicting short- and long-term mortality in breast cancer patients. Ann Surg

Cite this article as: Zhang Q, Yang Q, Weng Y, Huang Z, Chen R, Zhu Y, Dai K, Zhang S, Jiang S, Yu K. Neutrophilto-lymphocyte ratio correlates with prognosis and response to chemotherapy in patients with Non-M3 de novo acute myeloid leukemia. Transl Cancer Res 2021;10(2):1013-1024. doi: 10.21037/ tcr-20-2179
Oncol 2012;19:217-24.

27. Jung J, Lee H, Yun T, et al. Prognostic role of the neutrophil-to-lymphocyte ratio in patients with primary central nervous system lymphoma. Oncotarget 2017;8:74975-86.

28. Li Y, Li H, Li W, et al. Pretreatment neutrophil/ lymphocyte ratio but not platelet/lymphocyte ratio has a prognostic impact in multiple myeloma. J Clin Lab Anal 2017;31:e22107.

29. Coussens LM, Werb Z. Inflammation and cancer. Nature 2002;420:860-7.

30. Hanahan D, Weinberg RA. Hallmarks of cancer: the next generation. Cell 2011;144:646-74.

31. Grivennikov SI, Greten FR, Karin M. Immunity, inflammation, and cancer. Cell 2010;140:883-99.

32. Salazar-Onfray F, Lopez MN, Mendoza-Naranjo A. Paradoxical effects of cytokines in tumor immune surveillance and tumor immune escape. Cytokine Growth Factor Rev 2007;18:171-82.

33. Showalter A, Limaye A, Oyer JL, et al. Cytokines in immunogenic cell death: Applications for cancer immunotherapy. Cytokine 2017;97:123-32.

34. Dunn GP, Old LJ, Schreiber RD. The immunobiology of cancer immunosurveillance and immunoediting. Immunity 2004;21:137-48.

35. Le Jeune C, Bertoli S, Elhamri M, et al. Initial absolute lymphocyte count as a prognostic factor for outcome in acute myeloid leukemia. Leuk Lymphoma 2014;55:855-62.

36. Byrd JC, Mrozek K, Dodge RK, et al. Pretreatment cytogenetic abnormalities are predictive of induction success, cumulative incidence of relapse, and overall survival in adult patients with de novo acute myeloid leukemia: results from Cancer and Leukemia Group B (CALGB 8461). Blood 2002;100:4325-36.

37. Palanisamy N. Chromosomal translocations in AML: detection and prognostic significance. Cancer Treat Res 2010;145:41-58. 